\title{
BIOMARKERS VERSUS DUPLEX ULTRASONOGRAPHY FOR EARLY DETECTION OF ACUTE KIDNEY INJURY IN DOGS: AN EXPERIMENTAL STUDY
}

\author{
Mohamed A. Donia, Naglaa A. Gomaa*, Mohamed Abdelmegeid, Midhat N. Nassif
}

Department of Animal Medicine, Faculty of Veterinary Medicine, Kafrelsheikh University, 33516, Egypt

*Corresponding author, E-mail: naglaagomaa505@yahoo.com

\begin{abstract}
Acute kidney injury (AKI) is a common problem in dogs and is associated with high mortality. Early recognition of AKI is lifesaving and could help in clinical management. The purpose of this experimental study was to identify the accurate biomarkers for early detection of $\mathrm{AKI}$ in dogs. Ten healthy mongrel dogs ( 7 females, 3 males) were used in a crossover experimental study. Cisplatin $(5 \mathrm{mg} / \mathrm{kg} \mathrm{BW})$ was used for induction of AKI. Serum urea and creatinine, as well as serum Sodium, Potassium, Calcium, Phosphorus, and Bicarbonate, were measured spectrophotometrically using commercial kits. However, urinary Neutrophil Gelatinase-Associated Lipocalin (NGAL) were determined using ELISA. Renal Resistive Index (RI) was calculated using Doppler ultrasound (duplex). All examinations were carried out on the same day of induction and for other 4 consecutive days. Post-mortem and histopathological examinations were carried out on two dogs. Using the repeated measure MANOVA and student T-test, the data were statistically analyzed. There were significant increases of both serum urea and creatinine beginning from the second day of infusion, while renal RI was significantly increase beginning from the third day of infusion. There are marked changes in serum electrolytes including hyperkalemia, hyperphosphatemia, hyponatremia with metabolic alkalosis. However, urinary NGAL showed a non- significant increase during the experiment. It can be concluded that renal RI can be used for early diagnosis of acute renal injury in dogs.
\end{abstract}

Key words: urinary biomarkers; renal resistive index; acute kidney injury; dogs

\section{Introduction}

Acute kidney injury is a common problem in dogs. It is costive either for diagnosis or for treatment and correlated with high mortality (1). The earlier diagnosis of such condition is regarded as lifesaving (2). There are many methods for detection of kidney injury such as estimation of glomerular filtration rate (GFR) (3). The routine technique used for detection of
GFR is to measure serum creatinine concentration, but it is relatively insensitive with a delayed release in serum (4).

Novel serum and urine biomarker that can differentiate between both acute and chronic glomerular and tubular renal injury is the Neutrophil Gelatinase-Associated Lipocalin (NGAL) (5). It is a derived protein which increases in plasma and urine in acute ongoing renal injury after few hours (6). In human, NGAL 
was investigated in cases of AKI secondary to cardiac surgery, contrast-induced nephropathy and in critical illnesses. It was reported that elevated NGAL concentrations are a marker for AKI in such cases (7). Urinary NGAL is more sensitive and specific for predicting AKI and it was superior to creatinine and other markers of renal diseases (8).

One of the most advanced methods for the detection of AKI is the Doppler-based renal resistive index (RI) measurement. This is a rapid and non-invasive investigative tool that was used for early detection of AKI in the intensive care unit patients (9). Moreover, it can differentiate transient from persistent AKI in critically ill patients $(10,11)$. Recently, it was reported that an increase in the renal RI is an accurate and sensitive tool for early prediction of AKI in rats (12).

This study aims to compare the efficiency of selected serum biochemical parameters and the renal RI for early diagnose of AKI in dogs and to determine the most accurate, rapid, and applicable method in clinical practice.

\section{Material and methods}

\section{Animals}

Ten healthy mongrel dogs ( 7 females, 3 males) were used in this a cross over experimental study. Their weight ranged from 8 to 20 $\mathrm{Kgs}$ and their age ranged from 9 to 15 months. They considered healthy based on physical, hematological, biochemical and ultrasonographic examinations, in addition to a urine analysis. They were housed restless in separate boxes with plenty of food and water. The dogs were used as a control and treated group. In the control group, each dog was infused with normal saline at a dose rate of $5 \mathrm{ml} / \mathrm{kg} \mathrm{BW}$. After two weeks, the same dogs were used as a treated group in which they were infused once with Cisplatin (Mylan, France) at a concentration of $5 \mathrm{mg} / \mathrm{kg}$ for induction of AKI. Physical, hematological, serum biochemical profiles, urine analysis, and ultrasonographic examinations were carried out on each dog of the treated group on the same day of induction and for other 4 consecutive days. All examinations were done after approval of the Ethics committee in Kafrelsheikh University.

\section{Samples}

Two blood samples were collected from each dog from the cephalic vein in the control and treated group. The first blood sample was about $5 \mathrm{ml}$ that collected in a heparinized test tube for hematological examination using Vet hematological analyzer (Mythic18, France). The second blood sample was collected in a plain test tube to obtain serum for biochemical analysis. The serum was collected by centrifugation of the clotted blood at $3000 \mathrm{rpm}$ for 10 min. It was examined for blood urea nitrogen (BUN) using the colorimetric method by (BTS302 Spectrophotometer) and (Urea Biosystem kit, Germany). Serum creatinine was estimated using the fixed time method by (BTS-302Spectrophotometer) and (Creatinine Biosystem kit, Germany). Some blood electrolytes such as Sodium, Potassium, Chloride, Calcium, Phosphorus and the Bicarbonate were measured in the serum using (Cobas C \& Cobas E, France).

Urine samples were assembled by cystocentesis under ultrasonographic guide from each dog. Urine was examined macroscopically for colour and consistency. The specific gravity was measured by a standard Refractometer. The chemical examination for urine samples was accomplished using (Combi screen urine strips, Germany). Urine samples were examined microscopically for the presence of pus, cells, casts, and others. The investigation of Neutrophil Gelatinase-Associated Lipocalin (NGAL) was carried out using Sandwich enzyme-linked immunosorbent assay ELISA- kit (Canine NGAL Elisa Kit (Bioporto ${ }^{\circledR}$ Diagnostic) (13).

\section{Ultrasonographic examination}

Ultrasonographic examination was performed on unsedated dogs in both groups for detection of any changes in the tissue architecture, kidneys dimensions using 2-D mode. Renal (RI) for each kidney was measured using Doppler ultrasound (duplex), (Mindray Z5 ultrasound machine, China), with a linear transducer 5-10 MHZ.A renal interlobar or arcuate 
artery was distinguished firstly by color Doppler then switched on the pulsed wave Doppler using a gate of $1.5 \mathrm{~mm}$ width over the selected artery. The obtained image showed the blood flow without any aliasing. Multiple pulses from one artery in three poles for each kidney right and left in each dog was recorded (14). The renal RI was measured atomically by the ultrasound machine from the selected artery.

\section{Post mortem examination}

It was performed on two dogs, one of them dead at the $5^{\text {th }}$ day of the experiment and the second has been euthanized by a large dose of anesthetics and kidney specimens were picked in formalin $4 \%$ for histopathological examination.

\section{Histopathology}

Kidneys were removed, fixed in $4 \%$ buffered paraformaldehyde, embedded in paraffin with usual manner, sections were about $2.5 \mathrm{~mm}$ thick), and stained with periodic acid-schiff and periodic acid-methenamine silver for histologic examination under light microscope (15).

\section{Statistical analysis}

A doubly repeated measure MANOVA was used to test the significant effect of cisplatin on the parameter; urea, creatinine, urinary NGAL, and the renal RI among the examined animals at $P$ value $\leq 0.05$. This followed by univariate analysis for each parameter to examine its significant difference per day. Student T-test was used for comparison between serum electrolytes before and after the drug infusion and $P$ value $\leq 0.05$. All analyses applied using SPSS version 23.0 (IBM, New York, NY, USA).

\section{Results}

The physical examination of examined animals revealed; non-significant changes in all digital body parameters such as body temperature, heart rate respiratory rate and pulse. The mucous membranes and superficial lymph nodes were normal. Only signs of vomiting and diarrhea appeared after half hour of the drug infusion in all dogs. A degree of lethargy was clear on 5 dogs that obviously increased with time.
The physical examination of the urine samples showed changes in its colour beginning from the $3^{\text {rd }}$ day of Cisplatin infusion in most dogs. The appearance of the urine changed gradually from the amber yellow colour to the turbid deep yellow then reddish bloody in two dogs. By chemical analysis, the urine samples showed traces of proteins and glucose beginning from the second day then increased gradually to three or four pluses at the end of the experiment using strips kits. The microscopical examination of urine sediments revealed a marked increase of epithelial cells, pus cells, RBCs, urate and phosphate crystals within the time of the experiment.

There were no obvious changes in the 2D ultrasonographic measurements between before and after the Cisplatin infusion. The renal dimensions (length, width, and height) were between (5.64-6.30 mm, 2.79-4 $\mathrm{mm}$ and 2.56-3.3 $\mathrm{mm})$, respectively. On the other hand, there was a significant increase in renal RI of the interlobar arteries beginning from $3^{\text {rd }}$ day after infusion at $P$ value $\leq 0.022$ as in table 1 and (Fig. $1)$.

Concerning the blood serum biochemical analysis (Table1), revealed a significant increase in both BUN and creatinine at $(P$-value $\leq 0.000$ and 0.002 ), respectively from the second day after cisplatin infusion. On the other hand, the urinary NGAL concentration showed non-significant during all the days of this study.

Regarding serum electrolyte analysis (Table 2) showed insignificant $(P=0.058)$ decrease in serum sodium and insignificant $(P=0.09)$ increase in the serum ionized calcium. While there was a significant increase in serum inorganic phosphorus, potassium, and bicarbonate ions at $(P \leq 0.01,0.01$ and 0.000$)$, respectively.

PM examination of the first dog revealed some congestion in both kidneys and intestine, while the second showed no abnormalities except slight congestion of kidneys (Fig. 2 A).

Histopathological examination showed numerous apoptotic renal epithelial cells and thickening of the glomerular membranes, periglomerular fibrosis and marked renal tubular damage (Fig. 2 B). 
Table 1 Serum urea, serum creatinine, urinary NGAL and renal RI (mean and slandered deviation) after intravenous infusion of Cisplatin in dogs

\begin{tabular}{|c|c|c|c|c|c|c|c|}
\hline & Control & \multicolumn{5}{|c|}{ Time post-infusion (day) } & $P$ value \\
\hline & & 1 & 2 & 3 & 4 & 5 & \\
\hline $\begin{array}{l}\text { Urea } \\
(\mathrm{mg} / \mathrm{dl})\end{array}$ & $24.25 \pm 5.56$ & $26 \pm 3.16$ & $37.25 \pm 2.17 * *$ & $81.25 \pm 31.19^{* *}$ & $119.25 \pm 46^{* *}$ & $327.5 \pm 54.65^{* *}$ & 0.001 \\
\hline $\begin{array}{l}\text { Creatinine } \\
(\mathrm{mg} / \mathrm{dl})\end{array}$ & $0.94 \pm 0.06$ & $0.91 \pm 0.07$ & $1.35 \pm 0.07 * *$ & $1.71 \pm 0.33 * *$ & $2.72 \pm 0.28 * *$ & $6.71 \pm 0.91^{* *}$ & 0.000 \\
\hline $\begin{array}{l}\text { Urinary } \\
\text { NGAL } \\
(\mathrm{ng} / \mathrm{ml})\end{array}$ & $0.82 \pm 0.25$ & $2.23 \pm 1.09$ & $1.67 \pm 0.94$ & $2.03 \pm 1.18$ & $2.17 \pm 0.97$ & $2.15 \pm 1.26$ & 0.131 \\
\hline Renal RI & $0.62 \pm 0.03$ & $0.62 \pm 0.02$ & $0.64 \pm 0.03$ & $0.68 \pm 0.03 * *$ & $0.7 \pm 0.03 * *$ & $0.74 \pm 0.04 * *$ & 0.001 \\
\hline
\end{tabular}

*Indicates a significant difference at different time points $(\mathrm{p}<0.05)$

**Indicates a significant difference at different time points $(\mathrm{p}<0.01)$

Table 2: Serum sodium, potassium, ionized calcium, phosphorus and bicarbonate (mean and slandered deviation) before and after $5^{\text {th }}$ day of intravenous infusion of Cisplatin in dogs

\begin{tabular}{|ll|l|l|}
\hline & & Before infusion & After infusion \\
\hline Sodium & $(\mathrm{mEq} / \mathrm{l})$ & $138 \pm 2.58$ & $126.25 \pm 12.61$ \\
\hline Potassium & $(\mathrm{mEq} / \mathrm{l})$ & $4.18 \pm 0.23$ & $5.65 \pm 0.9 *$ \\
\hline Ionized calcium & $(\mathrm{mg} / \mathrm{dl})$ & $4.78 \pm 0.25$ & $5.28 \pm 0.64$ \\
\hline Phosphorus & $(\mathrm{mg} / \mathrm{dl})$ & $4.98 \pm 0.13$ & $5.83 \pm 0.59^{*}$ \\
\hline $\begin{array}{l}\text { Bicarbonate } \\
\text { (mmol/L) }\end{array}$ & & $18.48 \pm 0.66$ & $30.5 \pm 4.20^{* *}$ \\
\hline
\end{tabular}

*Indicates a significant difference at $\mathrm{p}<0.05$

**Indicates a significant difference at $\mathrm{p}<0.01$

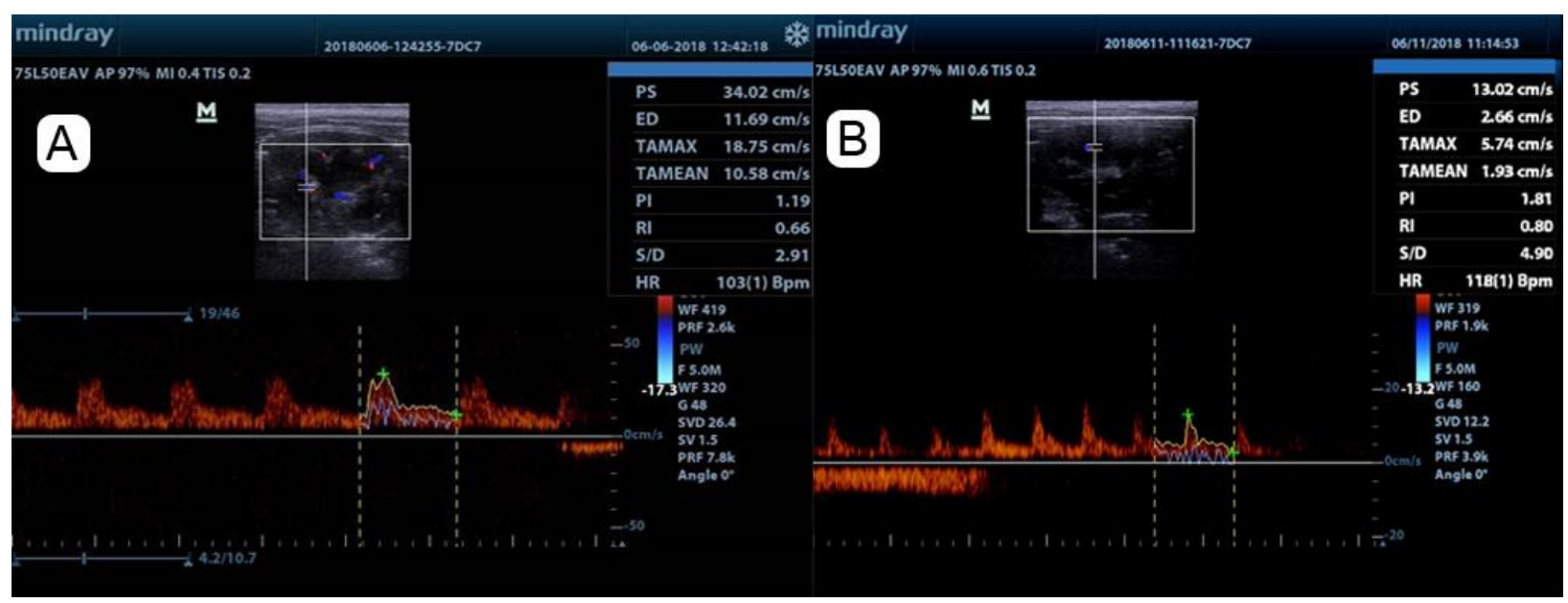

Figure 1: Pulsed wave Doppler ultrasonographic image of the interlobar artery before cisplatin infusion (A) and at the fifth day after the infusion (B) 


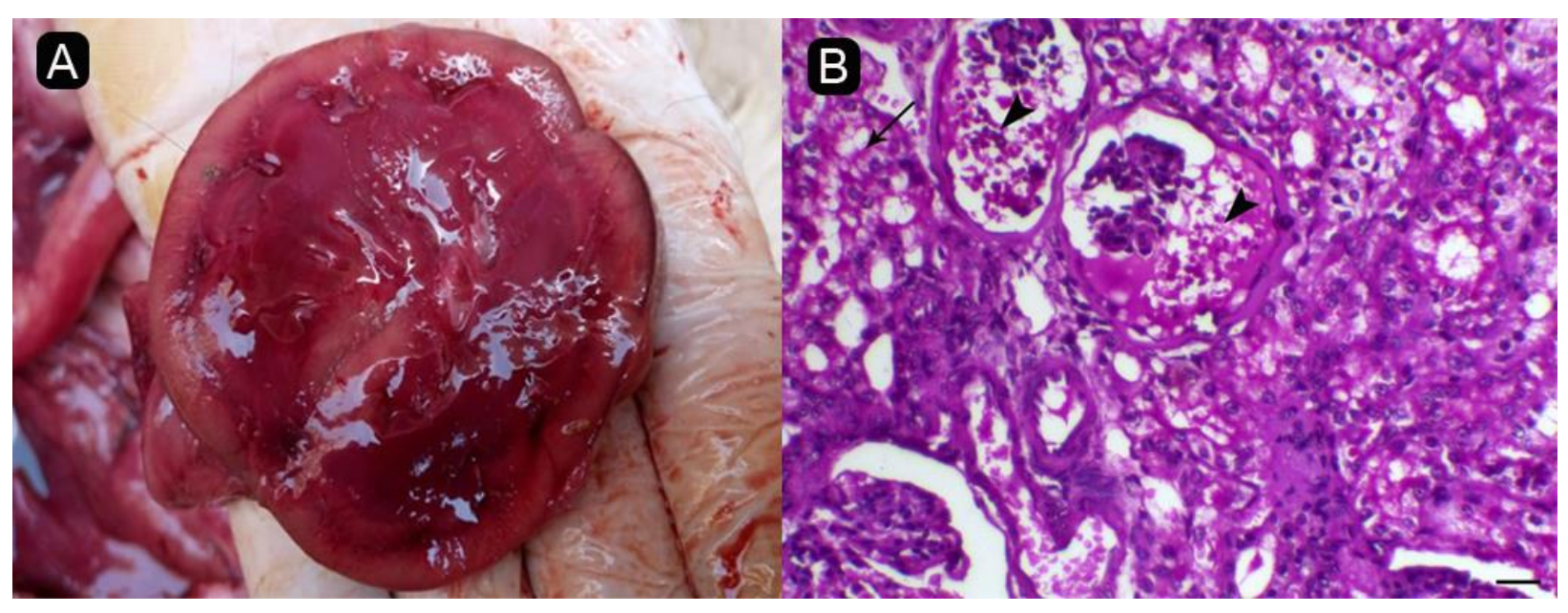

Figure 2: A sagittal section of the kidney showing congestion (A). Histopathological section of the kidney (B), shows marked necrotic changes with glomerular tufts which filled with eosinophilic materials (arrowhead) and marked degenerative and necrotic changes within the renal tubular epithelium (arrow), H\&E, bar $=40 \mu \mathrm{m}$

\section{Discussion}

In this study, acute renal injury was induced using cisplatin $5 \mathrm{mg} / \mathrm{kg}$ BW which is a widely used chemotherapeutic agent for different malignancies. It has a marked nephrotoxic effect as it causes renal tubular cell necrosis due to the presence of platinum in its structure $(16,17)$. Some of the treated dogs showed vomiting and diarrhea after infusion of the drug. This could be a side effect of the cisplatin due to its irritating effect on the mucosa of gastrointestinal tract $(18,19)$. Days after the infusion, there was ataxia or muscle weakness appeared on the dogs and it may be contributed to electrolytes disturbance and dehydration.

After two days of the infusion, urine samples revealed an obvious increase of glucose and this may due to the failure of reabsorption of the glucose from the proximal convoluted tubules (20). Beginning from the $3^{\text {rd }}$ day after infusion, there was a patent increase in proteins and RBCs in urine samples. This increase may be related to the decrease of glomerular filtration ate which follows the proximal tubular damage as an initial toxic effect of the cisplatin (17). After two days of infusion, there was a highly significant increase in serum urea level compared with the control group. However, serum creatinine was significantly increased after three days of the infusion and this may be attributed to the impaired kidney function (15). Although the estimation of urea and creatinine level in serum is routine work in the interpretation of kidney disease in the clinical practice, they have some limitations such as serum urea level may be increased in other abnormalities rather than kidney diseases such as liver dysfunction or increased dietary protein (21). It may also increase in cases of gastrointestinal bleeding as a consequence of increased absorption of nitrogenous compound (22). In addition, the magnitude of creatinine concentration cannot predict the origin and severity of renal dysfunction (23). Moreover, its concentration is reduced in sepsis and so limits its use as a marker of kidney injury in case of bacterial infections (24).

NGAL is a neutrophil-derived protein related to lipocalin protein family (25). Its expression is upregulated by injured epithelia of renal tubules, trachea, bronchi, and also increase in the acute infections and different types of neoplasia $(26,27)$. NGAL is markedly upregulated in damaged proximal tubules in order to induce re-epithelization (28). NGAL was reported as 
an early urinary biomarker for acute renal injury in dogs (29). Besides, it can be used as a real-time indicator of progressive kidney damage (30). In this study, NGAL was insignificantly increasing after the $5^{\text {th }}$ day of infusion of cisplatin. This insignificant increase of NGAL may be attributed to several causes such as the lower dose of cisplatin used in this study and the low sensitivity of the test in the detection of mild renal injury $(28,31)$. Therefore, the measurement of urinary NAGL is not efficient for the detection of mild kidney injury. Moreover, it increases in septic condition, so it gives a false positive result in septic cases (32).

Electrolyte disturbance usually occurs with kidney dysfunction especially those related to the renal tubules. The results of this study showed insignificant hyponatremia which may be attributed to reduced sodium conservation from damaged renal tubules (33). The significant hyperkalemia and hyperphosphatemia may have referred to the leakage of potassium and phosphate from the intracellular fluids to the extracellular fluids $(43,35)$. The highly significant increase of bicarbonate is unusual in such cases but, it may be due to vomition which develops an extra-renal cause of metabolic alkalosis. Alkalosis enhances calcium reabsorption in the distal nephron and this clarifies the insignificant increase in ionized calcium $(36,37)$.

Ultrasonography is very useful in the diagnosis of all aspects of renal diseases in clinical practice (38). In this study, each kidney was evaluated for any changes in echogenicity, length, width, height and volume by the $2 \mathrm{D}$ mode. There were non-significant changes neither in the echogenicity nor in kidney dimensions when compared with the control group (39). The Doppler ultrasonography can be used for assessing the renal hemodynamic which is very useful in the diagnosis of renal arteries and veins disorders such as thrombosis (40).

The renal RI was significantly $(p=0.022)$ increase beginning from the $3^{\text {rd }}$ day of the infusion. It may have referred to vasoconstriction resulting from renin release in response to the decreased blood flow in the renal arteries secondary to renal injury (41). This result coincided with other studies on dogs with interstitial nephritis or tubular degeneration (42). In the present study, the increase of RI was parallel with the increase of serum creatinine after the third day of infusion. In another study on mice, the renal RI precedes the serum creatinine in a diagnosis of AKI after cisplatin infusion (12). However, there are some limitations for using RI in the diagnosis of all kidney diseases because it is affected by other hemodynamic disorders rather than in kidney as well as the difficulty of measuring RI without sedation of dogs which affected on its measurement $(10,12)$.

The histopathological picture supported the clinical and biochemical findings which indicating tubular renal injury with secondary glomerular affection. This result was similar to that described in mice kidney after using cisplatin for induction of renal failure (43).

\section{Conclusion}

The results indicate that increasing renal RI go parallel with increasing serum urea and creatinine in dogs with AKI. Although renal RI alone is not sensitive for predicting the developing of AKI, the high increase of renal RI can be used as an early signal for Acute renal injury because of its high specificity.

\section{Conflict of interest}

None of the authors have any conflict of interest to declare.

\section{References}

1. Langston C. Acute uremia In Ettinger S,Feldman E, eds. Textbook of Veterinary Internal Medicine. Philadelphia: Saunders WB, 2010; 19552115.

2. Cowgill L, Langston C. Acute kidney insufficiency In Nephrology and Urology of small animals 2011;472-523.

3. Kerl M, Cook C. Glomerular filtration rate and renal scintigraphy. Clinical techniques in small animal practice $2005 ; 20: 31-8$.

4. Cobrin A, Blois S, Kruth S, et al. Biomarkers in the assessment of acute and chronic kidney diseases in the dog and cat. Journal of Small Animal Practice 2013; 54:647-55.

5. Urbschat A, Obermüller N, Haferkamp A. Biomarkers of kidney injury. Biomarkers 2011; 16 : S22-S30. 
6. Haase M, Haase-Fielitz A, Bellomo R, et al. Neutrophil gelatinase-associated lipocalin as a marker of acute renal disease. Current opinion in hematology 2011; 18:11-8.

7. Hirsch R, Dent C, Pfriem H, et al. NGAL is an early predictive biomarker of contrast-induced nephropathy in children. Pediatric Nephrology 2007; 22:2089.

8. Nickolas T, O'Rourke M, Yang J, et al. Sensitivity and specificity of a single emergency department measurement of urinary neutrophil gelatinaseassociated lipocalin for diagnosing acute kidney injury. Annals of internal medicine 2008; 148:810-9.

9. Darmon M, Schortgen F, Vargas F, et al. Diagnostic accuracy of Doppler renal resistive index for reversibility of acute kidney injury in critically ill patients. Intensive care medicine 2011; 37:6876.

10. Platt J, Rubin J, Ellis J. Acute renal failure: possible role of duplex Doppler US in distinction between acute prerenal failure and acute tubular necrosis. Radiology 1991; 179:419-23.

11. Schnell D, Deruddre S, Harrois A, et al. Renal resistive index better predicts the occurrence of acute kidney injury than cystatin C. Shock 2012;38:592-7.

12. Fisch S, Liao R, Hsiao L, et al. Early Detection of Drug-Induced Renal Hemodynamic Dysfunction Using Sonographic Technology in Rats. Journal of visualized experiments: JoVE 2016; 109:52409-4145

13. Steinbach S, Weis J, Schweighauser A, et al. Plasma and Urine Neutrophil Gelatinase-Associated Lipocalin (NGAL) in Dogs with Acute Kidney Injury or Chronic Kidney Disease. Journal of veterinary internal medicine 2014; 28:264-9.

14. Lin G, Cher T. Renal vascular resistance in normal children-a color Doppler study. Pediatric Nephrology1997; 11:182-5.

15. Abinaya A, Karu P, Karunakaran R, et al. Influence of age on blood biochemical profile of obese dogs. International Journal of Conservation Science 2018; 6:991-3.

16. Lim C, Han J, Kim S, et al. Effect of granulocyte colony-stimulating factor administration on renal regeneration after experimentally induced acute kidney injury in dogs. American journal of veterinary research 2016; 77:199-207.

17. KintzelP. Anticancer drug-induced kidney disorders. Drug safety2001; 24:19-38.

18. Sharma R, Tobin P, Clarke S. Management of chemotherapy-induced nausea, vomiting, oral mucositis, and diarrhea. The lancet oncology2005; 6:93-102.
19. Florea A, Büsselberg D. Cisplatin as an antitumor drug: cellular mechanisms of activity, drug resistance, and induced side effects. Cancers2011; 3:1351-71.

20. Bagshaw S, Langenberg C, Wan L, et al. A systematic review of urinary findings in experimental septic acute renal failure. Critical care medicine2007; 35:1592-8.

21. Sherlock S, Dooley J. Assessment of liver function In Diseases of the liver and biliary system: John Wiley \& Sons, 2008; 19-38.

22. Prause L, Grauer G. Association of gastrointestinal hemorrhage with increased blood urea nitrogen and BUN/creatinine ratio in dogs: a literature review and retrospective study. Veterinary clinical pathology1998; 27:107-11.

23. Perrone R, Madias N, Levey A. Serum creatinine as an index of renal function: new insights into old concepts. Clinical chemistry 1992; 38:1933-53.

24. Doi K, Yuen P, Eisner C, et al. Reduced production of creatinine limits its use as a marker of kidney injury in sepsis. Journal of the American Society of Nephrology 2009; 20:1217-21.

25. Flower D. The lipocalin protein family: structure and function. Biochemical journal.1996; 318:1-14.

26. Bolignano D, Donato V, Lacquaniti A, et al. Neutrophil gelatinase-associated lipocalin (NGAL) in human neoplasias: a new protein enters the scene. Cancer letters 2010; 288:10-6.

27. Fjaertoft G, Foucard T, Xu S, et al. Human neutrophil lipocalin (HNL) as a diagnostic tool in children with acute infections: a study of the kinetics. Acta Paediatrica2005; 94:661-6.

28. Mishra J, Mori K, Ma Q, et al. Neutrophil gelatinase-associated lipocalin: a novel early urinary biomarker for cisplatin nephrotoxicity. American journal of nephrology2004; 24:307-15.

29. Kai K, Yamaguchi T, Yoshimatsu Y, et al. Neutrophil gelatinase-associated lipocalin, a sensitive urinary biomarker of acute kidney injury in dogs receiving gentamicin. The Journal of toxicological sciences 2013; 38:269-77.

30. Mori K, Nakao K. Neutrophil gelatinase-associated lipocalin as the real-time indicator of active kidney damage. Kidney international 2007; 71:96770.

31. Steinbach S, Weis J, Schweighauser A, et al. Plasma and Urine Neutrophil Gelatinase-Associated Lipocalin (NGAL) in Dogs with Acute Kidney Injury or Chronic Kidney Disease. Journal of veterinary internal medicine2014; 28: 264-9. 
32. Yilmaz A, Sevketoglu E, Gedikbasi A, et al. Early prediction of urinary tract infection with urinary neutrophil gelatinase-associated lipocalin. Pediatric Nephrology 2009; 24:2387.

33. Denis C, Jadot V, Bouquegneau A, et al. The Case Acute renal failure and refractory hyponatremia. Kidney international2016; 90:713-4.

34. Tanrikut C, McDougal W. Acid-base and electrolyte disorders after urinary diversion. World journal of urology 2004; 22:168-71.

35. Haycock G. Management of acute and chronic renal failure in the newborn. Seminars in neonatology 2003; 325-34.

36. Jeon U, Pressure B. Kidney and calcium homeostasis. Electrolyte and Pressure, Blood 2008; 6:68-76.

37. Felsenfeld A, Levine B. Milk-alkali syndrome and the dynamics of calcium homeostasis. Clinical Journal of the American Society of Nephrology2006; 4:641-54.
38. O'Neill W. Sonographic evaluation of renal failure. American journal of kidney diseases 2000; 35:1021-38.

39. Adams W, Toal R, Breider M. Ultrasonographic findings in ethylene glycol (antifreeze) poisoning in a pregnant queen and 4 fetal kittens. Veterinary Radiology 1991; 32:60-2.

40. Ricci M, Lloyd D. Renal venous thrombosis in infants and children. Archives of Surgery1990; 125:1195-9.

41. Chang Y, Chan I, Cheng F, et al. Relationship between age, plasma renin activity, and renal resistive index in dogs. Veterinary radiology and ultrasound 2010; 51:335-7.

42. Morrow K, Salman M, Lappin M, et al. Comparison of the resistive index to clinical parameters in dogs with renal disease. Veterinary Radiology and Ultrasound 1996; 37:193-9.

43. Ramesh G, Reeves W. TNFR2-mediated apoptosis and necrosis in cisplatin-induced acute renal failure. American Journal of Physiology-Renal Physiology2003; 285: F610-F8. 